\title{
SINGULARITY FORMATION AND INSTABILITY IN THE UNSTEADY INVISCID AND VISCOUS PRANDTL EQUATIONS*
}

\author{
LAN HONG ${ }^{\dagger}$ AND JOHN K. HUNTER ${ }^{\ddagger}$
}

\begin{abstract}
We use the method of characteristics to prove the short-time existence of smooth solutions of the unsteady inviscid Prandtl equations, and present a simple explicit solution that forms a singularity in finite time. We give numerical and asymptotic solutions which indicate that this singularity persists for nonmonotone solutions of the the viscous Prandtl equations. We also solve the linearization of the inviscid Prandtl equations about a shear flow. We show that the resulting problem is weakly, but not strongly, well-posed, and that it has an unstable continuous spectrum when the shear flow has a critical point, in contrast with the behavior of the linearized Euler equations.
\end{abstract}

\section{Introduction}

The unsteady, two-dimensional Prandtl equations for the flow in an incompressible boundary layer are

$$
\begin{aligned}
& u_{t}+u u_{x}+v u_{y}+p_{x}^{\infty}=\nu u_{y y}, \\
& u_{x}+v_{y}=0 \\
& u(x, 0, t)=v(x, 0, t)=0, \\
& u(x, y, t) \rightarrow u^{\infty}(x, t) \quad \text { as } y \rightarrow+\infty, \\
& u(x, y, 0)=u_{0}(x, y) .
\end{aligned}
$$

Here, $-\infty<x<\infty$ and $y>0, u(x, y, t)$ and $v(x, y, t)$ are the $x$ and $y$ velocity components, respectively, $u_{0}(x, y)$ is given initial data, and $u^{\infty}(x, t)$ is a given external flow. The pressure $p^{\infty}(x, t)$ is related to $u^{\infty}(x, t)$ by

$$
p_{x}^{\infty}=-\left(u_{t}^{\infty}+u^{\infty} u_{x}^{\infty}\right) .
$$

It is remarkable that despite the importance and apparent simplicity of the unsteady Prandtl equations, very few rigorous results are known about them. For example, even the local existence of solutions with general smooth initial data remains an open question. One of the few known existence results is due to Oleinik [22], who proved that if the initial data satisfies the monotonicity condition $u_{0 y}>0$, then there is a unique local (either in space $x$ or time $t$ ) smooth solution. Under the same monotonicity condition, Xin and Zhang [32] have recently proved that there is a global weak solution, and they conjecture that this global weak solution is in fact smooth. Sammartino and Caflisch [26] used a Cauchy-Kowalewski argument to prove local existence without a monotonicity assumption, but with the very strong condition of analytic data. See [3], [6], [13], [23] for recent reviews of the Prandtl equations.

Smooth solutions of (1.1) do not, in general, exist globally in time. The formation of singularities was observed in numerical solutions of (1.1) by van Dommelen and Shen [10] for data corresponding to an impulsively started flow past a cylinder. A proof of the finite-time break down of smooth solutions of (1.1) with $u^{\infty}=p^{\infty}=0$ for suitable initial data was given by $\mathrm{E}$ and Engquist [12].

\footnotetext{
* Received: August 28, 2002; accepted: September 28, 2002.

$\dagger$ Department of Mathematics, University of California at Davis, Davis CA 95616, USA.

$\ddagger$ Department of Mathematics, University of California at Davis, Davis CA 95616, USA. Partially supported by the NSF under grant number DMS-0072343.
} 
The mathematical pathologies of the Prandtl equations, such as a possible illposedness or lack of strong well-posedness and the formation of singularities, reflect their well-known physical difficulties, including the inability to model boundary layer separation and upstream influence. These defects may be remedied in part by the use of triple-deck theories (see [28], [29] for introductions and further references). Nevertheless, in view of the fundamental nature of the Prandtl equations themselves, it is important to develop a complete understanding of their properties.

In particular, the lack of an adequate theory for the Prandtl equations makes it difficult to prove rigorously that the Prandtl boundary layer theory can be used to approximate the Navier-Stokes equations in the high Reynold's number limit under suitable assumptions (such as laminar flow and the absence of Rayleigh instabilities) even when boundary layer separation does not occur. The only case in which a proof is available is that of analytic data [27]. Although the use of laminar boundary layer theory is somewhat paradoxical, since boundary layer theory applies in a high Reynolds number limit when most rotational flows are turbulent [6], the rigorous justification of boundary layer theory remains a basic unresolved question in mathematical fluid mechanics.

One of the goals of this paper is to gain insight into some of the difficulties inherent in the Prandtl equations by a careful study of the inviscid Prandtl equations. As observed by van Dommelen [9], we can solve the inviscid Prandtl equations by the method of characteristics, and this enables us to obtain a great deal of explicit information about their solutions. In order to focus on the essential phenomena with a minimum of complications, we restrict attention to the pressureless Prandtl equations throughout most of this paper.

In Section 2, we show that there is a unique local smooth solution of the inviscid Prandtl equations for general smooth initial data. We also show that this solution is global for monotone initial data that is constant on the boundary. In the case of nonmonotone data, the local solution is, in general, less smooth in $x$ than the initial data. This loss of smoothness in the inviscid Prandtl equations makes it difficult to analyze the corresponding viscous equations. Nevertheless, it would be surprising if the introduction of viscosity were to destroy local existence for nonmonotone data.

In Section 3, we present a simple, explicit family of solutions of the inviscid Prandtl equations that develop singularities in finite time. These solutions provide a clear illustration of the mechanism of singularity formation, and the nature of the resulting singularities. We show that after singularities form there is a similar phenomenon to the singular delta-shocks that arise in hyperbolic conservation laws. In Section 4, we give numerical and asymptotic results which indicate that similar inviscid-type singularities form in nonmonotone solutions of the viscous Prandtl equations.

One approach to understanding the continuous dependence of solutions of the Prandtl equations on their initial data is to consider the linearization of the Prandtl equations about various solutions. Here, it is also useful to begin by studying the inviscid equations. The simplest nontrivial solution is that of a shear flow. In Section 5, we study the linearization of the inviscid Prandtl equations about a shear flow, and solve it explicitly. We show that the linearized equations are weakly, but not strongly, well-posed, owing to a loss of $x$-derivatives.

In Section 6, we use this solution to show that the linearization of the Prandtl equations about a shear flow has an unstable continuous spectrum when the shear profile has a critical point, but does not have an unstable point spectrum. This is in 
contrast with the linearization of the Euler equations about a shear flow, which may have an unstable point spectrum when the shear profile has an inflection point [11], but whose continuous spectrum is always stable (see [4], [8], [14], [25], [31]). We also present numerical solutions of the inviscid and viscous Prandtl equations with initial data corresponding to a perturbed shear flow. These solutions agree well with the linearized analysis.

It is easy to understand why the linearized Euler equations can have an unstable point spectrum although the linearized inviscid Prandtl equations do not: the unstable eigenfunctions of the linearized Euler equations depend on lengthscales that are of the same order of magnitude in the $x$ and $y$ directions, so they do not satisfy the scaling assumptions that lead to the inviscid Prandtl equations, and they are lost in the Prandtl limit. If such unstable modes are present, then one cannot expect the Prandtl equations to provide a good approximation of the corresponding Euler or NavierStokes equations, even though there may be no sign of singularities or instabilities in the Prandtl solutions, because the growth of the unstable modes due to a Rayleigh instability may lead to a rapid divergence of the Euler or Navier-Stokes solutions from the Prandtl solutions (see [6] for further discussion, [16] for an instability theorem, and [2] for numerical results). The qualitatively different behavior of the continuous spectra of the linearized Euler and inviscid Prandtl equations is more puzzling, and we do not have a good explanation for it.

\section{Existence for the inviscid Prandtl equations}

We consider the unsteady, inviscid Prandtl equations with zero pressure:

$$
\begin{aligned}
& u_{t}+u u_{x}+v u_{y}=0, \\
& u_{x}+v_{y}=0, \\
& v(x, 0, t)=0, \\
& u(x, y, 0)=u_{0}(x, y) .
\end{aligned}
$$

In the inviscid case, we do not impose boundary conditions on $u$ at $y=0$ or $y=+\infty$.

Solutions of (2.1)-(2.4) provide formal asymptotic approximations to solutions of the incompressible Euler equations that satisfy the usual boundary layer scalings, in which the $x$-velocity component is $O(1)$, the $y$-velocity component is $O(\epsilon)$, the lengthscale of variations in $x$ is $O(1)$, and the lengthscale of variations in $y$ is $O(\epsilon)$, where $\epsilon \ll 1$. For work on related inviscid equations (the "homogeneous hydrostatic" equations), see [1], [15].

Van Dommelen [9] observed that the inviscid Prandtl equations can be solved exactly by the method of characteristics. We use this fact to prove the following local existence result, not given in [9]. The main point of this result is that no monotonicity condition is required on the initial data in order to obtain local existence.

Proposition 2.1. Let $u_{0}(x, y)$ be smooth initial data such that

$$
t_{*}=-\left[\inf _{x \in \mathbb{R}, y>0} u_{0 x}(x, y)\right]^{-1}>0 .
$$

Then there exists a unique smooth solution of (2.1)-(2.4) in $0 \leq t<t_{*}$, given by

$$
\begin{aligned}
& u(x, y, t)=u_{0}(\xi(x, \eta(x, y, t), t), \eta(x, y, t)), \\
& v(x, y, t)=-\int_{0}^{\eta(x, y, t)} \frac{u_{0 x}(\xi(x, Z, t), Z)}{\left[1+t u_{0 x}(\xi(x, Z, t), Z)\right]^{2}} d Z,
\end{aligned}
$$


where $\xi(x, Y, t)$ and $\eta(x, y, t)$ are the solutions of

$$
\begin{aligned}
& x=\xi+t u_{0}(\xi, Y) \\
& y=\int_{0}^{\eta} \frac{d Z}{1+t u_{0 x}(\xi(x, Z, t), Z)} .
\end{aligned}
$$

Proof. First suppose that $(u, v)$ is a smooth solution. We introduce characteristic coordinates $X, Y$, where $x=x(X, Y, t), y=y(X, Y, t)$ satisfy

$$
\begin{array}{ll}
x_{t}=u, & x(X, Y, 0)=X, \\
y_{t}=v, & y(X, Y, 0)=Y .
\end{array}
$$

It follows from (2.1) and (2.4) that

$$
u=u_{0}(X, Y)
$$

Hence, from (2.9),

$$
x=X+t u_{0}(X, Y) .
$$

Since $X_{x}=\left[1+t u_{0 x}\right]^{-1}>0$ when $0 \leq t<t_{*}$, we may solve this equation for $X=\xi(x, Y, t)$, where $\xi$ is defined by $(2.7)$.

The incompressibility condition (2.2) implies that the Jacobian of the transformation between spatial and characteristic coordinates is constant, so

$$
x_{X} y_{Y}-x_{Y} y_{X}=1 .
$$

Using (2.12) in this equation, we get

$$
\left(1+t u_{0 X}\right) y_{Y}-t u_{0 Y} y_{X}=1 .
$$

The characteristics of this equation are $x=$ constant, or $X=\xi(x, Y, t)$. Moreover, since $v=0$ when $y=0$, the boundary $y=0$ is a particle path, so $y=0$ when $Y=0$. Integrating (2.13) along characteristics, we get

$$
y=\int_{0}^{Y} \frac{d Z}{1+t u_{0 x}(\xi(x, Z, t), Z)} .
$$

Differentiation of $y$ with respect to $t$, with $(X, Y)$ held fixed, gives (2.6). Thus, any smooth solution is of the form stated.

Conversely, suppose $0 \leq t<t_{*}$. Then, since $X_{x}=\left[1+t u_{0 x}\right]^{-1}>0$, equation (2.12) is invertible to give $X=\xi(x, Y, t)$, where $\xi$ is defined by (2.7); and, since $Y_{y}=1+t u_{0 x}>0$, equation (2.14) is invertible to give $Y=\eta(x, y, t)$, where $\eta$ is uniquely defined by (2.8). One can then check directly that (2.5)-(2.8) defines a smooth solution of (2.1)-(2.4).

It is clear from (2.8) that there may be a loss of $x$-derivatives in the inviscid solution. As an explicit example, one can use the method of characteristics to compute the solution of (2.1)-(2.4) for the initial data

$$
u_{0}(x, y)=\frac{1}{4} x|x|+y^{2} .
$$


There is a jump discontinuity in $u_{0 x x}$ at $x=0$, and a loss of strict monotonicity on the boundary, since $u_{0 y}=0$ when $y=0$. We will not write out the complete solution here, but one can show that for $t>0$

$$
u(x, y, t)=\widetilde{u}(x, y, t) \quad \text { in } x \leq 0,
$$

where the smooth function $\widetilde{u}$ is given by

$$
\widetilde{u}(x, y, t)=\frac{2}{t^{2}}\left\{\sqrt{1-t x} \cosh (t y)-1+\frac{1}{2} t x\right\} \quad x<\frac{1}{t},
$$

and

$$
u(x, y, t)-\widetilde{u}(x, y, t) \sim \frac{4}{3 t^{2}}(t x)^{3 / 2} \sinh (t y) \quad \text { as } x \rightarrow 0^{+} .
$$

Thus, although $u_{0} \in C^{1,1}$, we only have $u \in C^{1,1 / 2}$ for $t>0$. We investigate this loss of smoothness further in the context of the linearized Prandtl equations in Section (5).

For monotone initial data, we may also solve the inviscid equations by use of the Crocco transformation.

Proposition 2.2. Let $u_{0}(x, y)$ be smooth initial data such that $u_{0 y}(x, y)>0$, there exists a constant $C$ such that

$$
\left|u_{0 x}\right| \leq C, \quad\left|u_{0 x y}\right| \leq C, \quad\left|u_{0 y y}\right| \leq C\left|u_{0 y}\right|,
$$

and

$$
t_{*}=-\left[\inf _{x \in \mathbb{R}} u_{0 x}(x, 0)\right]^{-1}>0 .
$$

Then there exists a unique smooth solution of (2.1)-(2.4) in $0 \leq t<t_{*}$,

Proof. If $u_{0 y}>0$, then the Crocco transformation,

$$
w(x, u, t)=u_{y}(x, y, t),
$$

reduces the inviscid Prandtl equations to the advection equation

$$
w_{t}+u w_{x}=0 .
$$

The solution is

$$
w(x, u, t)=w_{0}(x-u t, u)
$$

where

$$
w_{0}\left(x, u_{0}(x, y)\right)=u_{0 y}(x, y)
$$

This solution provides an ODE in $y$ for $u$,

$$
u_{y}(x, y, t)=w_{0}(x-u t, u)
$$

which has a global solution in $y$ for any given boundary data

$$
u(x, 0, t)=u_{b}(x, t)
$$


if the right-hand side is a globally Lipschitz function of $u$. Using (2.16), we compute that

$$
\left.\partial_{u} w_{0}(x-u t, u)\right|_{u=u_{0}}=-t u_{0 x y}+\left\{1+t u_{0 x}\right\} \frac{u_{0 y y}}{u_{0 y}} .
$$

Thus, the conditions in (2.15) are sufficient to ensure that $\partial_{u} w_{0}(x-u t, u)$ is bounded so $w_{0}(x-u t, u)$ is globally Lipschitz in $u$. (Of course, a solution for $u$ may exist under other conditions as well.)

Setting $y=0$ in the inviscid equations, when $v=0$, we obtain a Burgers equation for the boundary data $u_{b}$,

$$
\begin{aligned}
& u_{b t}+u_{b} u_{b x}=0, \\
& u_{b}(x, 0)=u_{0}(x, 0) .
\end{aligned}
$$

This IVP has a smooth solution in $0 \leq t<t_{*}$, and hence there is a unique smooth solution of the inviscid Prandtl equations in the same time interval.

In particular, if $u_{0}(x, 0)=$ constant, then $u_{b}$ is identically constant, and this solution is a global smooth solution. On the other hand, if $u_{0 x}(x, 0)<0$ at some point, then $u_{x}$ blows up on the boundary in finite time.

\section{Singularity formation in the inviscid Prandtl equations}

A global smooth solution of the inviscid Prandtl equations does not exist for general initial data, and a singularity may form in finite time. Physically, the singularity is caused by the compression of the fluid in the $x$-direction by nonlinear advection. When the fluid is bounded by a wall at $y=0$, this generates a jet in the $y$-direction. In the Euler equations, this compression creates a pressure gradient in the $y$-direction which opposes the compression of the fluid. In the Prandtl equations, the pressure gradient in the $y$-direction is zero, and there is no force to prevent the compression of fluid elements to zero length in $x$.

The next proposition gives a simple, exact "wavy shear flow" solution of the inviscid Prandtl equations that provides a clear illustration of the formation of a singularity.

Proposition 3.1. Let $U(y)$ and $\beta_{0}(x)$ be smooth functions. The solution of the inviscid Prandtl equations (2.1)-(2.4) with the initial data

$$
u_{0}(x, y)=U\left(y+\beta_{0}(x)\right)
$$

is given by

$$
\begin{aligned}
& u(x, y, t)=U(y+\beta(x, t)), \\
& v(x, y, t)=-\beta_{t}(x, t)-U(y+\beta(x, t)) \beta_{x}(x, t),
\end{aligned}
$$

where $\beta(x, t)$ is the solution of

$$
\begin{aligned}
& \beta_{t}+U(\beta) \beta_{x}=0, \\
& \beta(x, 0)=\beta_{0}(x) .
\end{aligned}
$$

If

$$
t_{*}=-\left[\inf _{x \in \mathbb{R}}\left\{U_{y}\left(\beta_{0}(x)\right) \beta_{0 x}(x)\right\}\right]^{-1}>0,
$$


then $u_{x}$ and $v$ blow up as $t \uparrow t_{*}$.

Proof. It is straightforward to check that (3.2)-(3.3) satisfies (2.1)-(2.2) for any smooth functions $U(y)$ and $\beta(x, t)$. Imposing the boundary condition (2.3) on (3.3), we obtain (3.4). Moreover, (3.2) satisfies the initial condition (2.4), (3.1) when $\beta$ satisfies (3.5). The derivative $\beta_{x}$ of the solution of (3.4)-(3.5) blows up at time $t_{*}$, and then $u_{x}$ and $v$ also blow up.

We may also obtain this solution from the general solution given in the previous proposition. According to the method of characteristics, the solution is

$$
\begin{aligned}
& u(x, y, t)=U\left(Y+\beta_{0}(X)\right), \\
& x=X+t U\left(Y+\beta_{0}(X)\right), \\
& y=\int_{0}^{Y} \frac{d Z}{1+t \beta_{0 x}(X(x, Z, t)) U^{\prime}\left(Z+\beta_{0}(X(x, Z, t))\right)} .
\end{aligned}
$$

We make the change of variable

$$
W(x, Z, t)=Z+\beta_{0}(X(x, Z, t))
$$

in the integral for $y$, and find that

$$
y=\int_{0}^{Y} d W=W(x, Y, t)-W(x, 0, t)=Y+\beta_{0}(X)-\beta_{0}(X(x, 0, t)) .
$$

We have $\beta_{0}(X(x, 0, t))=\beta(x, t)$, where $\beta(x, t)$ is the solution of $(3.4)-(3.5)$, so $Y+$ $\beta_{0}(X)=y+\beta(x, t)$, and $u=U(y+\beta(x, t))$, in agreement with (3.2).

Once singularities form, the inviscid Prandtl equations presumably no longer provide asymptotic approximations of the Euler equations, whose solutions remain smooth in two space dimensions. Nevertheless, we may ask the question whether or not it is possible to extend smooth solutions of the inviscid Prandtl equations by suitably defined weak solutions after they form singularities.

One way to extend the solution given in Proposition (3.1) past the blow-up time is to rewrite (3.4) in the conservation form,

$$
\beta_{t}+F(\beta)_{x}=0,
$$

where $F_{y}(y)=U(y)$ (so that $F$ is the streamfunction associated with the shear flow $U$ ), choose $\beta$ to be a weak solution of (3.7), and define $u, v$ by (3.2)-(3.3). However, some care is required to interpret the resulting functions as weak solutions of the Prandtl equations. If we write (2.1) in the conservation form

$$
u_{t}+\left(u^{2}\right)_{x}+(u v)_{y}=0,
$$

then, once $\beta$ becomes discontinuous, the $y$-flux $u v$ is formally a product of a stepfunction and a delta-distribution, so it is not defined in the usual distributional sense. This is similar to what happens for singular delta-shocks in conservation laws, such as those for a pressureless gas; see [18], [30]. Moreover, it is not clear what admissibility criterion should be used to select weak solutions of (3.7).

In Figure 3.1, we illustrate the behavior of this solution by showing a numerical solution of the inviscid Prandtl problem (2.1)-(2.4) with the initial data

$$
u_{0}(x, y)=y+\sin 2 \pi x .
$$


(a)
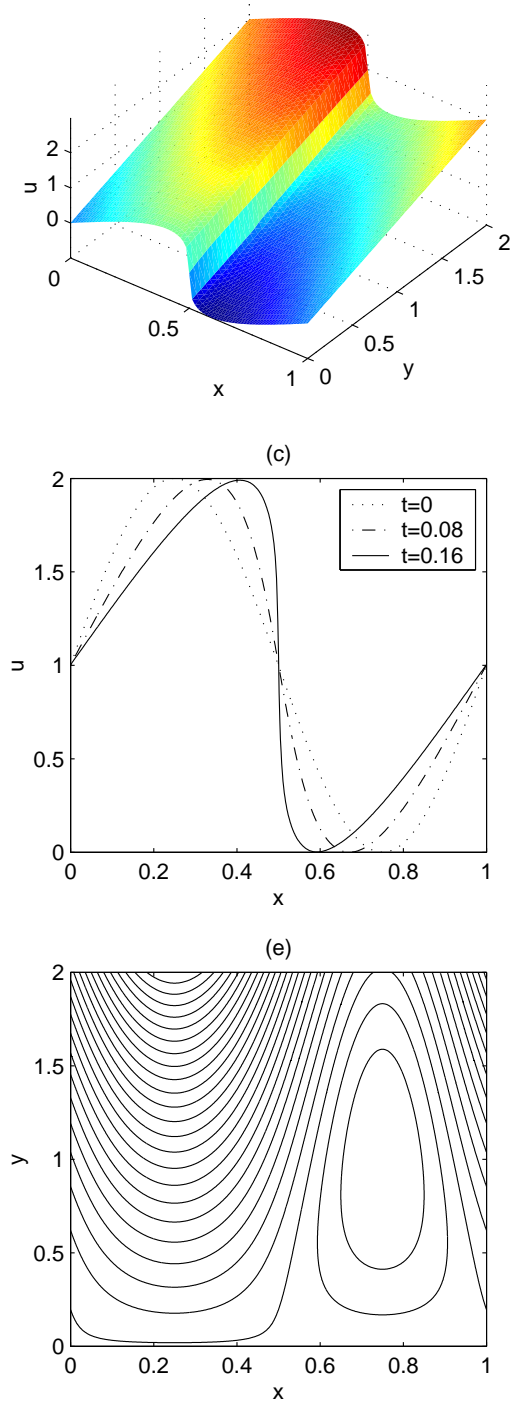

(b)
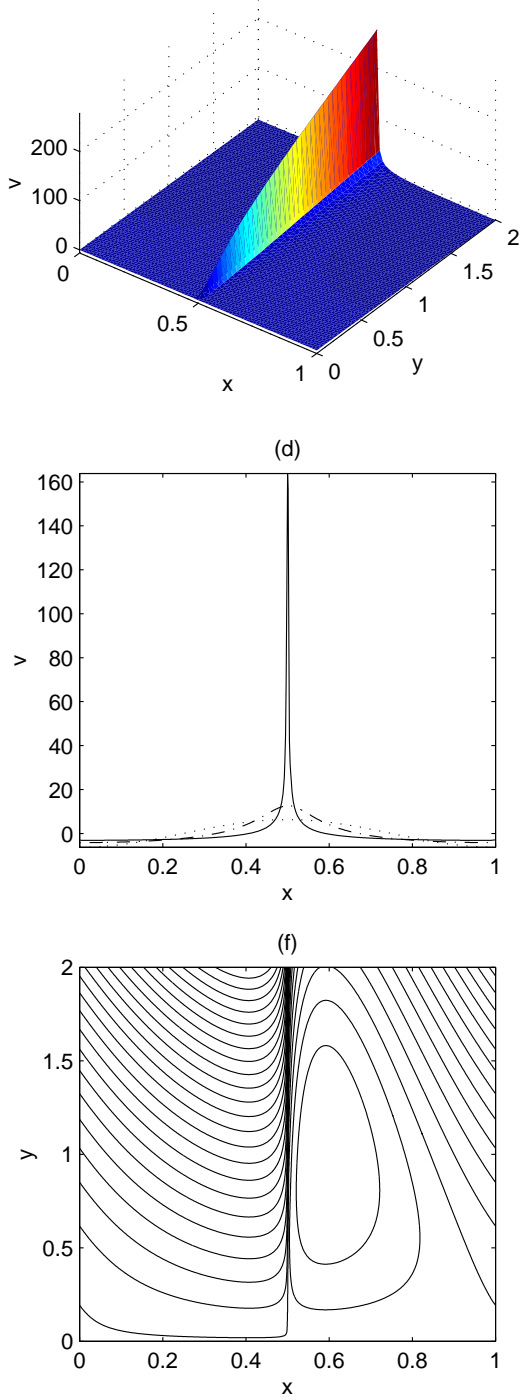

FIG. 3.1. A numerical solution of the inviscid Prandtl equations (2.1)-(2.4) with initial data (3.9): (a) surface plot of $u(x, y, t)$ at $t=0.16$; (b) surface plot of $v(x, y, t)$ at $t=0.16$; (c) plot of $u(x, y, t)$ versus $x$ at $y=1$ showing the formation of a shock; (d) plot of $v(x, y, t)$ versus $x$ at $y=1$ showing the formation of a singularity; (e) streamlines at $t=0 ;(f)$ streamlines at $t=0.16$.

This solution corresponds to a linear profile $U(y)=y$, and according to the exact solution $\beta(x, t)$ satisfies the inviscid Burgers equation

$$
\beta_{t}+\beta \beta_{x}=0, \quad \beta(x, 0)=\sin 2 \pi x,
$$

with breaking time $t_{*}=1 /(2 \pi) \approx 0.16$.

In the numerical computations, we introduce a finite domain $0 \leq x \leq 1,0 \leq y \leq 2$, with periodic boundary conditions in $x$, and use a uniform grid. We rewrite the 
inviscid Prandtl equations in conservation form (3.8), and discretize the resulting PDE explicitly in time. To obtain a numerical $x$-flux for $u^{2}$, we use a flux-limited method, with an Engquist-Osher flux as a first-order flux, a Lax-Wendroff flux as a second order flux, and a min-mod flux limiter. We use a Lax-Wendroff flux for the $y$-flux $u v$, and compute $v$ from $u$ by integration of (2.2), discretized using central differences. More details about the numerical scheme are given in [17].

We see the development of an apparent numerical $\delta$-function singularity in $v(x$, $y, t)$ at $x \approx 0.50$ and $t \approx 0.16$, corresponding to a "jet" of fluid going off to $y=+\infty$, in agreement with the analytical solution. After the singularity forms, the numerical solution for $u$ contains a stationary "shock," and the numerical solution for $v$ contains a stationary spike.

\section{Singularity formation in the viscous Prandtl equations}

In this section, we first present a generalization of the singular solution of the inviscid Prandtl equations that satisfies the viscous Prandtl equations. This solution satisfies the no-flow boundary condition at $y=0$, but does not satisfy the no-slip condition. When the viscosity is small, we use the method of matched asymptotic expansions to construct an asymptotic solution of the viscous Prandtl equations that satisfies the no-slip condition, and we use numerical computations to examine the formation of singularities. The numerical results indicate that the inclusion of $y$-viscosity typically does not prevent the singularity formation demonstrated in Proposition (3.1) for the inviscid Prandtl equations, unless the viscous solution is monotone.

We consider the pressureless viscous Prandtl equations

$$
\begin{aligned}
& u_{t}+u u_{x}+v u_{y}=\nu u_{y y}, \\
& u_{x}+v_{y}=0 .
\end{aligned}
$$

If $W(y, t)$ is any solution of the heat equation,

$$
W_{t}=\nu W_{y y}
$$

and $\beta(x, t)$ is an arbitrary smooth function, then

$$
\begin{aligned}
& u(x, y, t)=W(y+\beta(x, t), t), \\
& v(x, y, t)=-\beta_{t}(x, t)-W(y+\beta(x, t), t) \beta_{x}(x, t),
\end{aligned}
$$

satisfies (4.1)-(4.2). This solution follows from the solutions given in [21], although it is not written out explicitly there, nor is it used to study singularity formation in the initial-boundary value problem. It may be obtained by an application of the following symmetry transformation of the Prandtl equations

$$
x \mapsto x, \quad y \mapsto y+\beta, \quad u \mapsto u, \quad v \mapsto v+\beta_{t}+u \beta_{x},
$$

where $\beta(x, t)$ is an arbitrary function, to the diffusing shear flow solution

$$
u=W(y, t), \quad v=0 .
$$

See [20] for further exact solutions of the Prandtl equations.

If $W$ and $\beta$ satisfy

$$
W(y, 0)=U(y), \quad \beta(x, 0)=\beta_{0}(x),
$$


then (4.4) satisfies the initial condition

$$
u(x, y, 0)=U\left(y+\beta_{0}(x)\right) .
$$

The solution (4.5) satisfies the no-flow boundary condition

$$
v(x, 0, t)=0
$$

if $\beta$ is a solution of the time-dependent PDE

$$
\beta_{t}+W(\beta, t) \beta_{x}=0 .
$$

The derivative $\beta_{x}$ blows up along a characteristic at time $t_{*}$ if

$$
\int_{0}^{t_{*}} W_{y}\left(\beta_{0}, t\right) d t=-\frac{1}{\beta_{0 x}} .
$$

Therefore, provided that $W_{y}$ does not decay too rapidly in time, (4.10) has the same kind of blow up behavior as the time-independent equation (3.4).

In the viscous case, we also have to impose the no-slip condition

$$
u(x, 0, t)=0,
$$

which is not satisfied by (4.4) except in trivial cases. When $\nu$ is small, we expect that a boundary layer will form near the wall $y=0$, and that the above exact solution will provide an asymptotic approximation as $\nu \rightarrow 0$ to the solution outside the boundary layer, at least before it becomes singular. At leading order in $\nu$, and for $t=O(1)$, we have from (4.3) and (4.7) that $W(y, t) \sim U(y)$. Thus, the leading order outer solution is identical to the inviscid solution given in Proposition (3.1).

We consider the viscous Prandtl equations (1.1) as $\nu \rightarrow 0$. Using the method of matched asymptotic expansions, we find that the width of the boundary layer is of the order $\sqrt{\nu}$. The leading order outer solution

$$
u \sim u^{*}(x, y, t), \quad v \sim v^{*}(x, y, t),
$$

satisfies the inviscid Prandtl equations,

$$
\begin{aligned}
& u_{t}^{*}+u^{*} u_{x}^{*}+v^{*} u_{y}^{*}+p_{x}^{\infty}=0, \\
& u_{x}^{*}+v_{y}^{*}=0, \\
& v^{*}(x, 0, t)=0 \\
& u^{*}(x, y, 0)=u_{0}(x, y),
\end{aligned}
$$

when $y \gg \sqrt{\nu}$. The leading order inner solution

$$
u \sim \tilde{u}(\tilde{x}, \tilde{y}, \tilde{t}), \quad v \sim \sqrt{\nu} \tilde{v}(\tilde{x}, \tilde{y}, \tilde{t}), \quad \tilde{x}=x, \quad \tilde{y}=\frac{y}{\sqrt{\nu}}, \quad \tilde{t}=t,
$$

satisfies the full boundary layer equations,

$$
\begin{aligned}
& \tilde{u}_{\tilde{t}}+\tilde{u} \tilde{u}_{\tilde{x}}+\tilde{v} \tilde{u}_{\tilde{y}}+p_{x}^{\infty}=\tilde{u}_{\tilde{y} \tilde{y}}, \\
& \tilde{u}_{\tilde{x}}+\tilde{v}_{\tilde{y}}=0, \\
& \tilde{u}(\tilde{x}, 0, \tilde{t})=v(\tilde{x}, 0, \tilde{t})=0, \\
& \tilde{u}(\tilde{x}, \tilde{y}, \tilde{t}) \sim u^{*}(\tilde{x}, 0, \tilde{t}) \\
& \tilde{u}(\tilde{x}, \tilde{y}, 0)=u_{0}(\tilde{x}, 0) .
\end{aligned}
$$
as $\tilde{y} \rightarrow+\infty$ 
(a)

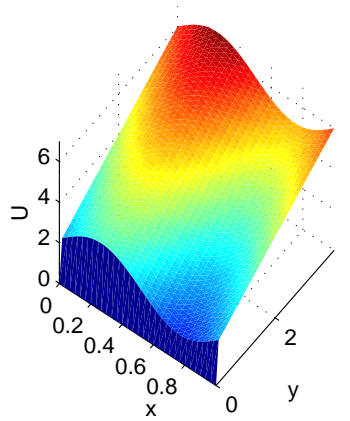

(d)
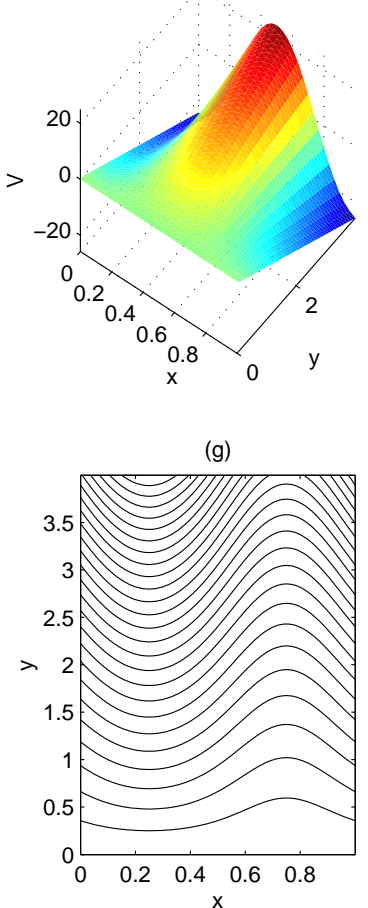

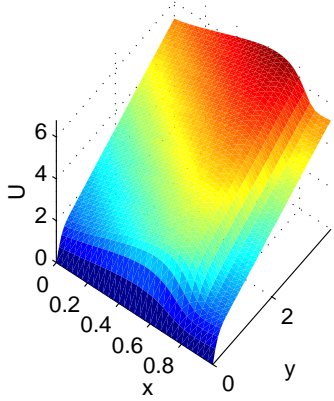

(e)

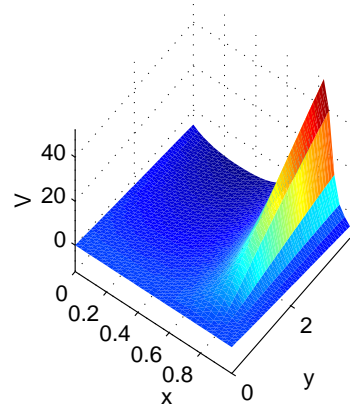

(h)

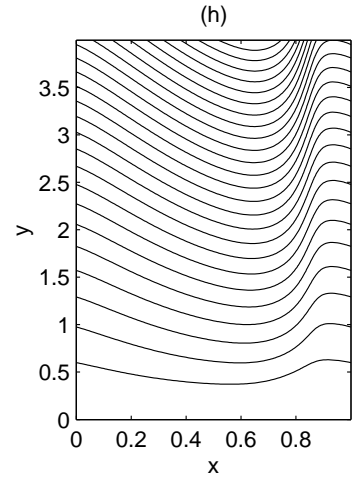

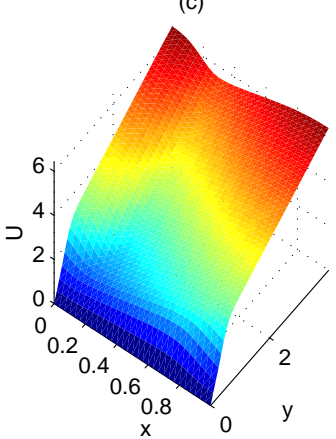

FIG. 4.1. A numerical solution of the viscous Prandtl equations (1.1) for $\nu=0.1$, with $p^{\infty}=0$, the initial data (4.12), and the boundary condition (4.13): (a)-(c) surface plots of $u(x, y, t)$ at $t=0.0,0.15,0.3 ;(d)-(f)$ surface plots of $v(x, y, t)$ at $t=0.0,0.15,0.3 ;(g)-(i)$ streamlines at $t=$ $0.0,0.15,0.3$.

when $y=O(\sqrt{\nu})$.

In general, this matched asymptotic solution breaks down when the outer solution becomes singular. It follows from the inner equations that

$$
\tilde{v}(\tilde{x}, \tilde{y}, \tilde{t}) \sim-\tilde{y} u_{x}^{*}(\tilde{x}, 0, \tilde{t}) \quad \text { as } \tilde{y} \rightarrow+\infty .
$$

Thus, when a singularity forms in the outer solution and $u_{x}^{*} \rightarrow-\infty$, the inner $\tilde{y}$ velocity $\tilde{v}$ becomes large and positive. This creates a "jet" from the inner layer into 
the outer layer, which couples the inner and outer solutions. It is then inconsistent to impose the no flow condition $v^{*}=0$ on the outer solution at $y=0$. Numerical computations show that in some cases this coupling can prevent the formation of a singularity in the outer solution.

In Figures 4.1-4.2, we show a solution of (1.1) with $p^{\infty}=0, \nu=0.1$, and the initial

(a)

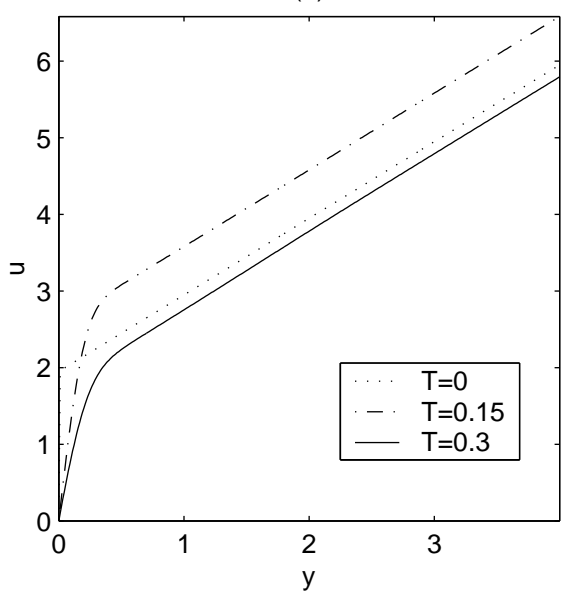

(c)

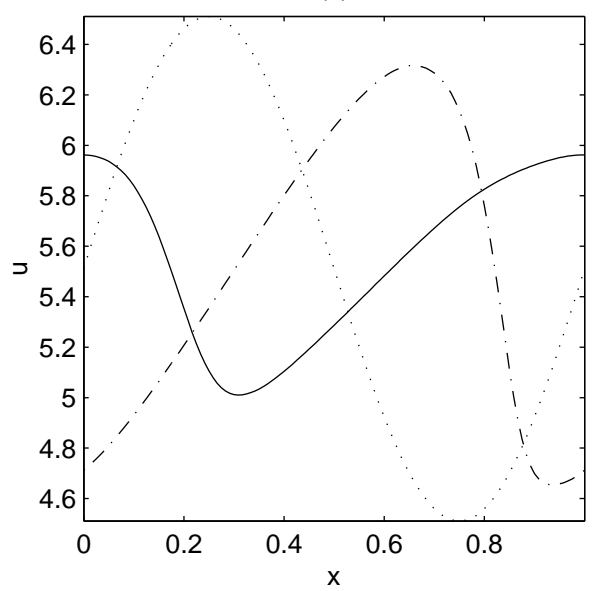

(b)

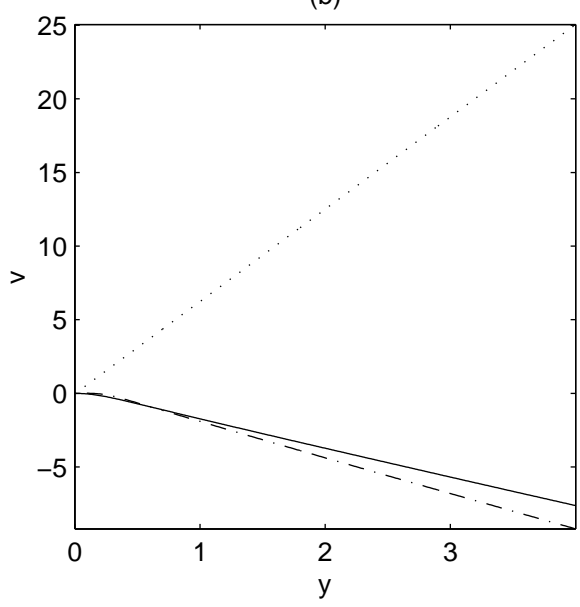

(d)

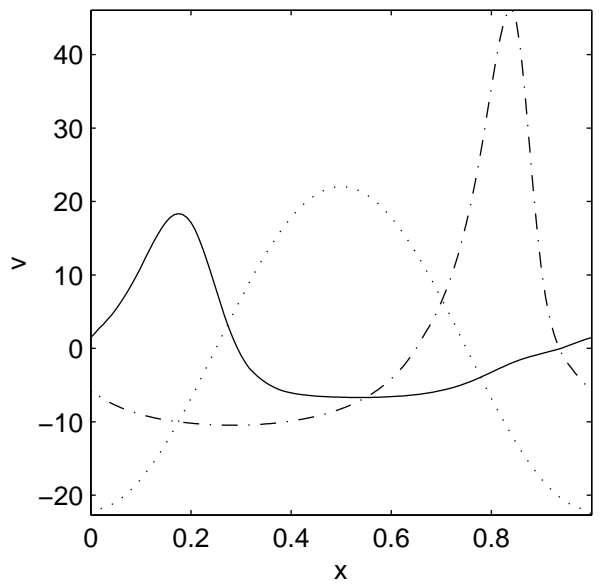

FIG. 4.2. Plots of $u$ and $v$ for the numerical solution of the viscous Prandtl equations shown in Figure 4.1: (a) $u(x, y, t)$ versus $y$ at $x=0.5$; (b) $v(x, y, t)$ versus $y$ at $x=0.5$; (c) $u(x, y, t)$ versus $x$ at $y=3.5 ;(d) v(x, y, t)$ versus $x$ at $y=3.5$.

data

$$
u_{0}(x, y)=2+y+\sin 2 \pi x .
$$

We impose the boundary condition

$$
u_{y}(x, y, t) \rightarrow 1 \quad \text { as } y \rightarrow+\infty
$$


instead of the boundary condition $u \rightarrow u^{\infty}$. (Similar results are observed for solutions that approach a given velocity at infinity.) The numerical scheme is the same as the one used for the inviscid equations, except that we discretize the viscous term $u_{y y}$ implicitly in time, and solve a tridiagonal system in $y$ to update $u$ [17].

In this case, we see the formation of a boundary layer near the wall $y=0$ and a jet with relatively large values of $v$, as predicted by the matched asymptotic solution;

(a)

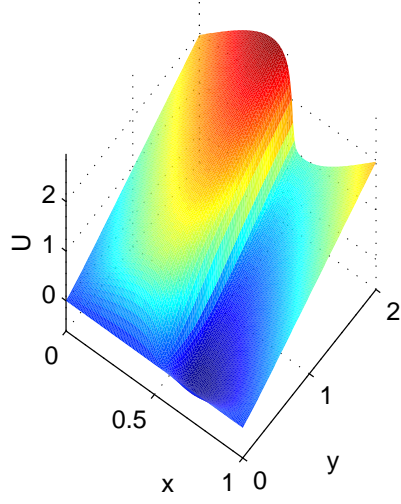

(d)

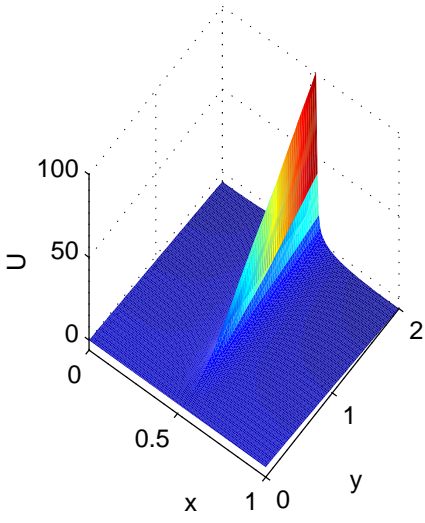

(b)

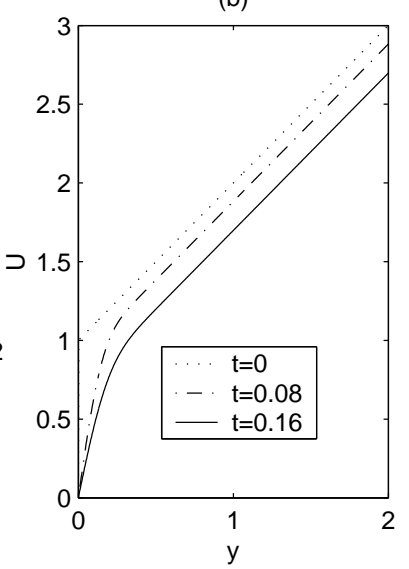

(e)

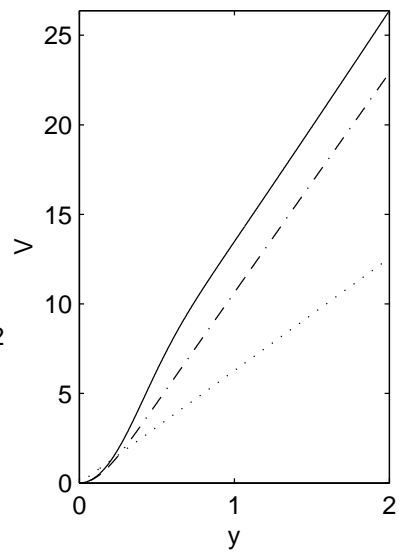

(c)

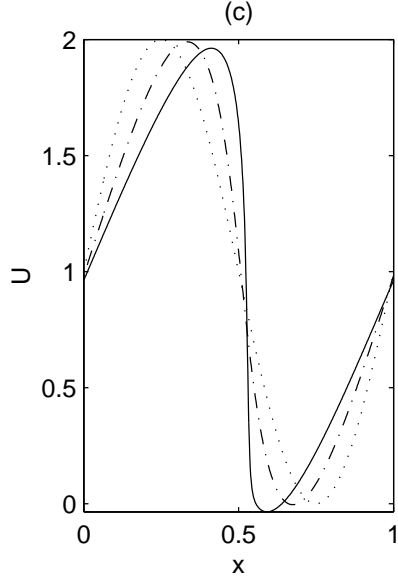

(f)

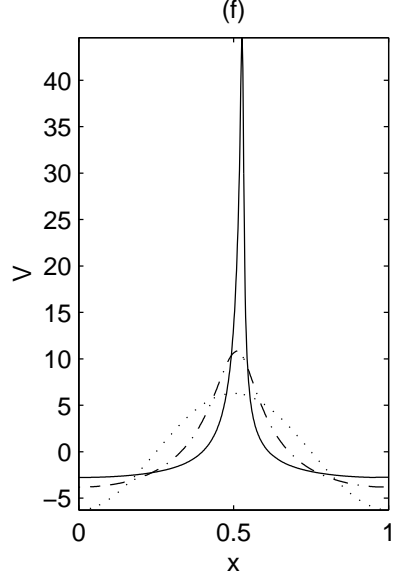

FIG. 4.3. A numerical solution of the viscous Prandtl equations (1.1) for $\nu=0.1$, with $p^{\infty}=0$, the initial data (3.9), and the boundary condition (4.13): (a) surface plot of $u(x, y, t)$ at $t=0.16$; (b) plots of $u(x, y, t)$ versus $y$ at $x=0.5$ showing the formation of a boundary layer; (c) plots of $u(x, y, t)$ versus $x$ at $y=1$ showing the formation of a shock; (d) surface plot of $v(x, y, t)$ at $t=0.16$; (e) plots of $v(x, y, t)$ versus $y$ at $x=0.5 ;(f)$ plots of $v(x, y, t)$ versus $x$ at $y=1$ showing the formation of a singularity.

but then $v$ decays, and there appears to be a global smooth solution, even though the solution of the corresponding inviscid problem does not remain smooth. For $t>0$, the viscous solution for $u$ is monotone in $y$, so these numerical results are consistent 
with the global existence result of Xin and Zhang [32] for monotone initial data.

In the nonmonotone case, however, we typically observe the formation an inviscidtype singularity in the numerical solution. In Figure 4.3, we show a solution of (1.1) with $p^{\infty}=0, \nu=0.1$, and the same initial data as in (3.9),

$$
u_{0}(x, y)=y+\sin 2 \pi x .
$$

(a)

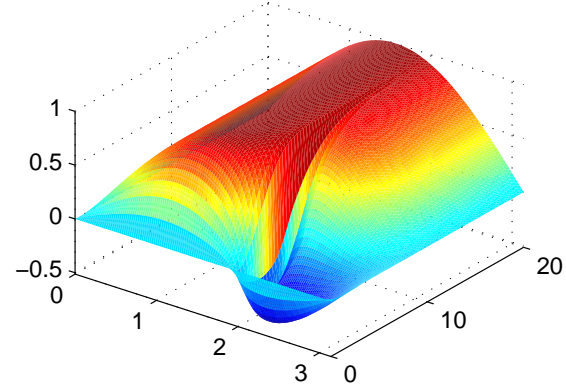

(c)

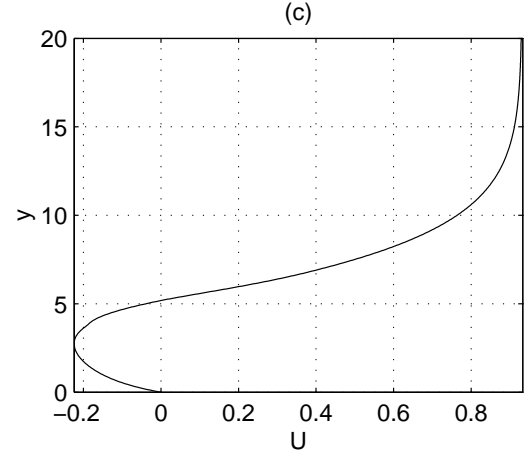

(b)

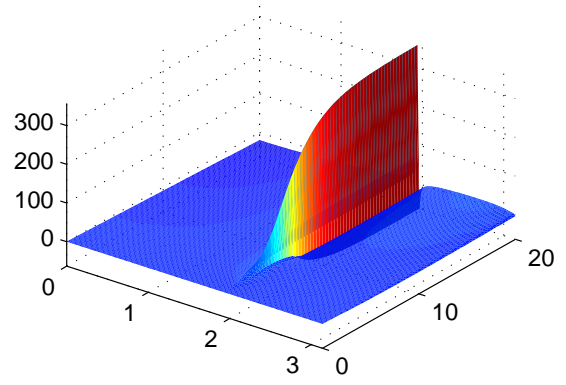

(d)

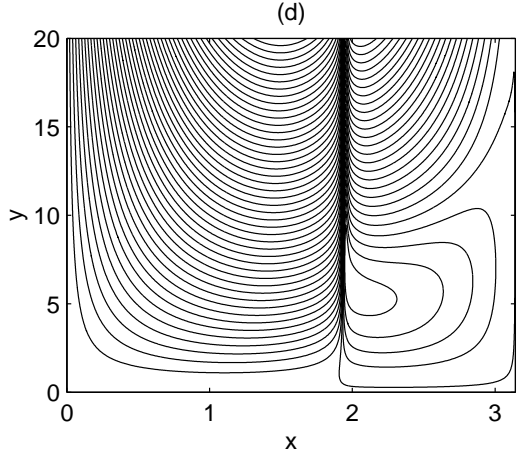

FIG. 4.4. A numerical solution of the viscous Prandtl equations (1.1) with data (4.14), corresponding to flow past a cylinder started impulsively from rest at the singularity formation time $t=3.0$ : (a) surface plot of $u(x, y, t)$; (b) surface plot of $v(x, y, t)$; (c) profile of $u$ versus $y$ at $x=1.94$; (d) streamlines.

The result shows the formation of an apparent singularity at $x \approx 0.50, t \approx 0.16$, as for the inviscid solution.

This inviscid mechanism of singularity formation appears to be rather typical. In Figure 4.4, we show a numerical solution of the cylinder problem studied by van Dommelen and Shen [10], which consists of (1.1) with the initial and boundary data

$$
u_{0}=\sin x, \quad u^{\infty}=\sin x, \quad 0 \leq x \leq \pi .
$$

We see a jet-like singularity form near the wall $y=0$ at $x \approx 1.94, t \approx 3.0$, similar to the one in the previous solution. For larger values of $y$, the streamlines "cross" the jet, and there is a flow of fluid out to infinity on the left of the jet, and in from infinity on the right of the jet. Our values for the location and time of the formation of a singularity are in excellent agreement with those of van Dommelen and Shen [10], who found $x \approx 1.942, t \approx 3.0$ using a Lagrangian scheme. (See also [5], [7], [24].) 
5. The linearization of the inviscid Prandtl equations at a shear flow In this section and the next, we study the well-posedness and stability of the linearization of the inviscid Prandtl equations about a shear flow solution $u=U(y)$, $v=0$. The linearization of $(2.1)-(2.4)$ is

$$
\begin{aligned}
& u_{t}+U u_{x}+U_{y} v=0, \\
& u_{x}+v_{y}=0, \\
& v(x, 0, t)=0, \\
& u(x, y, 0)=u_{0}(x, y) .
\end{aligned}
$$

Introducing a stream-function $\psi(x, y, t)$ such that

$$
u=\psi_{y}, \quad v=-\psi_{x}
$$

we may write (5.1) as

$$
\begin{aligned}
& \psi_{y t}+U \psi_{x y}-U_{y} \psi_{x}=0 \\
& \psi(x, 0, t)=0 \\
& \psi(x, y, 0)=\psi_{0}(x, y)
\end{aligned}
$$

where $\psi_{0}(x, y)=\int_{0}^{y} u_{0} d y$.

We may solve this equation in a standard way by the use of Fourier-Laplace transforms. The result suggests a quicker way of integrating (5.3) directly.

Proposition 5.1. If $U(y)$ and $u_{0}(x, y)$ are smooth functions, then the solution of (5.3) is given by

$$
\begin{aligned}
\psi(x, y, t)=\int_{0}^{y} & \left\{u_{0}(x-U(z) t, z)\right. \\
& \left.+t[U(y)-U(z)] u_{0 x}(x-U(z) t, z)\right\} d z,
\end{aligned}
$$

where $u_{0}=\psi_{0 y}$. The corresponding solution of (5.1) is given by

$$
\begin{gathered}
u(x, y, t)=u_{0}(x-U(y) t, y)+t U_{y}(y) \int_{0}^{y} u_{0 x}(x-U(z) t, z) d z \\
v(x, y, t)=-\int_{0}^{y}\left\{u_{0 x}(x-U(z) t, z)\right. \\
\left.+t[U(y)-U(z)] u_{0 x x}(x-U(z) t, z)\right\} d z .
\end{gathered}
$$

Proof. We introduce "Lagrangian" variables

$$
X=x-U(y) t, \quad Y=y, \quad T=t .
$$

The corresponding "Lagrangian" and "Eulerian" partial derivatives are related by

$$
\partial_{X}=\partial_{x}, \quad \partial_{Y}=\partial_{y}+t U_{y} \partial_{x}, \quad \partial_{T}=\partial_{t}+U \partial_{x}
$$

We also define a Lagrangian streamfunction $\Psi$ by

$$
\psi_{y}=\Psi_{Y}
$$


where $\Psi=0$ when $Y=0$. We will show that $\Psi$ satisfies (5.12) below, which we can integrate immediately.

We write the PDE in (5.3) as

$$
\partial_{T} \psi_{y}=U_{y} \psi_{x}
$$

Multiplying this equation by $T$, rearranging the result, and using (5.7), we find that

$$
\partial_{T}\left(T \psi_{y}\right)=\psi_{Y}
$$

Using (5.8) in the left-hand side of (5.10), and integrating the result with respect to $Y$, we obtain

$$
\partial_{T}(T \Psi)=\psi
$$

The function of integration is zero because both $\psi$ and $\Psi$ vanish when $Y=0$.

Using (5.8) and (5.11) in the left-and right-hand sides of (5.9), respectively, and rewriting the result with the help of 5.7 , we find that $\Psi$ satisfies

$$
\partial_{T} \Psi_{y}=0 .
$$

Integrating this equation, and imposing the initial and boundary conditions, we get that

$$
\Psi(x, y, t)=\int_{0}^{y} u_{0}(x-U(z) t, z) d z .
$$

Using this result in (5.11), and simplifying, we get the solution for $\psi$ in (5.4). The corresponding expressions for $u$ and $v$ follow from (5.2) and (5.4).

The solution (5.5) shows that there is a "loss" of $x$-derivatives in going from the initial data to the solution. Consequently, (5.1) is weakly well-posed, but not strongly well-posed. Specifically, let $\|\cdot\|$ be any translation-invariant $x$-norm, for example the $L_{x}^{2}$-norm

$$
\|u\|(y, t)=\left(\int_{-\infty}^{\infty}|u(x, y, t)|^{2} d x\right)^{1 / 2} .
$$

Then (5.5) immediately implies the following estimate.

Proposition 5.2. Let $u(x, y, t)$ satisfy (5.1), where $U(y)$ and $u_{0}(x, y)$ are smooth functions such that $\left\|u_{0}\right\|(y),\left\|u_{0 x}\right\|(y)$ are finite. Then

$$
\|u\|(y, t) \leq\left\|u_{0}\right\|(y)+t\left|U_{y}(y)\right| \int_{0}^{y}\left\|u_{0 x}\right\|(z) d z .
$$

In view of this estimate, a natural space in which to consider (5.1) is the space $X$ of functions $u: \mathbb{R} \times[0,1] \rightarrow \mathbb{C}$ such that

$$
y \mapsto u(\cdot, y) \in C\left([0,1] ; L_{x}^{2}(\mathbb{R})\right)
$$

with norm

$$
\|u\|_{X}=\sup _{0 \leq y \leq 1}\left(\int|u(x, y)|^{2} d x\right)^{1 / 2} .
$$


Here, we restrict $y$ to a compact interval, which we take to be $[0,1]$ for definiteness. We may write (5.1) abstractly as

$$
u_{t}=A u,
$$

where $A: D(A) \subset X \rightarrow X$ is the closed operator whose action on smooth functions is given by

$$
A=-U \partial_{x}+U_{y} \partial_{y}^{-1} \partial_{x}, \quad \partial_{y}^{-1}=\int_{0}^{y}
$$

By making a Galilean transformation in $x$, we may assume that $U(y)>0$ without loss of generality. In that case, we have the following estimate.

Proposition 5.3. Suppose that $U:[0,1] \rightarrow \mathbb{R}^{+}$is a smooth function which does not vanish. If $\lambda \in \mathbb{C}$ is not purely imaginary, then $\lambda$ belongs to the resolvent set of $A$ and

$$
\left\|(\lambda I-A)^{-1}\right\|_{X} \leq \frac{1}{|\operatorname{Re} \lambda|}+\frac{C}{|\lambda|-|\operatorname{Im} \lambda|},
$$

where

$$
C=\frac{1}{2} \sup _{0 \leq y \leq 1}\left\{\left|U_{y}(y)\right| \int_{0}^{y} \frac{d z}{|U(z)|}\right\}
$$

Proof. We consider the equation

$$
\lambda u-A u=f
$$

for smooth functions $f$ and $u$ that are rapidly decaying in $x$. Fourier transforming with respect to $x$, we obtain that

$$
(\lambda+i k U) \widehat{u}-i k U_{y} \partial_{y}^{-1} \widehat{u}=\widehat{f}
$$

where

$$
\widehat{u}(k, y)=\frac{1}{\sqrt{2 \pi}} \int_{-\infty}^{\infty} u(x, y) e^{-i k x} d x
$$

Solving (5.14), we find that

$$
\widehat{u}=\frac{\widehat{f}}{\lambda+i k U}+i k U_{y} \partial_{y}^{-1}\left[\frac{\widehat{f}}{(\lambda+i k U)^{2}}\right] .
$$

Estimating the right-hand side of this equation, we obtain

$$
|\widehat{u}| \leq \frac{|\widehat{f}|}{|\operatorname{Re} \lambda|}+\frac{\left|U_{y}\right|}{2(|\lambda|-|\operatorname{Im} \lambda|)} \partial_{y}^{-1}\left[\frac{|\widehat{f}|}{|U|}\right] .
$$

The result then follows from Parseval's theorem.

This resolvent estimate is not sufficient to imply that $A$ generates a strongly continuous group in $X$, and it is clear from (5.5) that it does not unless $U$ is constant. 
The spectrum of $A$ is continuous, and consists of the imaginary axis. For $k \in \mathbb{R}$ and $0 \leq c<1$, we define

$$
u_{k, c}(x, y)=U_{y}(y) H(y-c) e^{i k x}
$$

where $H$ is the step function

$$
H(y)= \begin{cases}1 & \text { if } y>0 \\ 0 & \text { if } y<0\end{cases}
$$

Then $u_{k, c}$ is a generalized eigenfunction of $A$, which formally satisfies

$$
A u_{k, c}=\lambda_{k, c} u_{k, c}, \quad \lambda_{k, c}=-i k U(c) .
$$

Hence, $-i k U(c)$ belongs to the approximate spectrum of $A$.

If we consider $A$ as acting on functions $u: \mathbb{T} \times[0,1] \rightarrow \mathbb{C}$ that are periodic in $x$, where $\mathbb{T}=\mathbb{R} / \mathbb{Z}$ is the torus, then $\lambda_{2 \pi n, c}$ belongs to the point spectrum of $A$ when $n \in \mathbb{Z}$ and $c=0$ or $U_{y}(c)=0$. The point spectrum of $A$ is embedded in a continuous spectrum, and we show in the next section that these modes dominate the large-time asymptotics of the solution.

\section{Linearized stability of shear flows}

In this section, we show that the shear flow solution $u=U(y)$ of the inviscid Prandtl equation is linearly unstable when $U$ has a critical point. Solutions grow algebraically in time, corresponding to an unstable continuous spectrum.

Proposition 6.1. Suppose that $u$ is the solution of (5.1), where $U$ is a smooth function and $u_{0}$ is smooth initial data that decays rapidly in $x$. If $U$ has no critical points, then the $L_{x}^{2}$-norm of $u$ in (5.13) is uniformly bounded in $t$, and

$$
\|u\|(y, t) \sim\left\|u_{0}\right\|(0) \frac{\left|U_{y}(y)\right|}{\left|U_{y}(0)\right|} \quad \text { as } t \rightarrow \infty .
$$

If $U$ has a single, nondegenerate critical point at $y=c>0$, then for $y>c$

$$
\|u\|(y, t) \sim \sqrt{2 \pi t}\left\|u_{0}\right\|_{1 / 2}(c) \frac{\left|U_{y}(y)\right|}{\sqrt{\left|U_{y y}(c)\right|}} \quad \text { as } t \rightarrow \infty,
$$

where

$$
\left\|u_{0}\right\|_{1 / 2}(y)=\left(\int_{\mathbb{R}}\left|\partial_{x}^{1 / 2} u_{0}(x, y)\right|^{2} d x\right)^{1 / 2}
$$

Proof. Fourier transforming (5.4) with respect to $x$, we find that the Fourier transform $\widehat{\psi}$ with respect to $x$ of the solution $\psi$ of (5.3), is given by

$$
\widehat{\psi}(k, y, t)=\int_{0}^{y} \widehat{u}_{0}(k, z)\{1+i k t[U(y)-U(z)]\} e^{-i k U(z) t} d z .
$$

If $U$ is a smooth function with no critical points, then an integration by parts in (6.3) shows that $\widehat{\psi}$ is bounded uniformly in time, and

$$
\widehat{\psi}(k, y, t) \sim \widehat{u}_{0}(k, 0)\left[\frac{U(y)-U(0)}{U_{y}(0)}\right] e^{-i k U(0) t} \quad \text { as } k t \rightarrow \infty .
$$


Differentiating this result with respect to $y$ and using Parseval's identity, we get (6.1). The corresponding solution for $u$ is a superposition of the generalized eigenfunctions $u_{k, 0}$ in (5.15).

If $U(y)$ has a nondegenerate critical point at $y=c>0$, then an application of the method of stationary phase to (6.3) implies that, when $y>c$,

$$
\widehat{\psi}(k, y, t) \sim i \sqrt{2 \pi k t} \widehat{u}_{0}(k, c)\left[\frac{U(y)-U(c)}{\sqrt{\left|U_{y y}(c)\right|}}\right] e^{-i k t U(c)-i \sigma \pi / 4} \quad \text { as } k t \rightarrow \infty,
$$

where $\sigma=\operatorname{sgn} U_{y y}(c)$. Differentiating this result with respect to $y$ and using Parseval's identity, we get (6.2). The corresponding solution for $u$ is a superposition of the generalized eigenfunctions $u_{k, c}$.

Thus, when $U$ has a nondegenerate critical point, the solution of (5.1) grows like $\sqrt{t}$. The growth is faster if $U$ has a degenerate critical point. For example, if $U$ is constant in some interval, then we get linear growth in $t$, as in the estimate in Proposition (5.2).

The solution for $u$ corresponding to (6.4) is proportional to $U_{y}$, so it may be interpreted an infinitesimal translation of the unperturbed shear flow in the $y$-direction. The mechanism of the instability is therefore that $v$ grows, and advects the shear flow (see Figure 6.1 below). The $L_{x}^{2}$-norm of the "vorticity" $\omega=u_{y}$ also grows in time. This growth is the result of the advection of vorticity into the region from $y=+\infty$.

There is a connection between the existence of an instability and the failure of strong well-posedness. The linearized equations (5.1) are invariant under the scaling transformations

$$
x \mapsto \lambda x, \quad y \mapsto y, \quad t \mapsto \lambda t, \quad u \mapsto u, \quad v \mapsto \frac{v}{\lambda} .
$$

As a result, $\widehat{u}(k, y, t)$ depends only on the product $k t$, and we have a family of solutions of (5.3) of the form

$$
u(x, y, t ; k)=\widetilde{u}(y, k t) e^{i k x} .
$$

Thus, growth in $t$ implies growth in $k$, and hence to a loss of $x$-derivatives. Since the growth in time is algebraic, the problem is weakly well-posed, but not ill-posed with respect to Sobolev norms (as it would be if there were an unstable point spectrum with exponential growth in time).

The weak well-posedness of (5.1) means that one has to be careful in determining its stability and well-posedness by the use of approaches that are not based on the solution of the initial value problem. For example, if one "freezes" coefficients in (5.1), and looks for Fourier mode solutions

$$
u(x, y, t)=\widehat{u} e^{i k x+i \ell y-i \omega t}, \quad v(x, y, t)=\widehat{v} e^{i k x+i \ell y-i \omega t}
$$

of the "frozen" equations, then one finds that, for $\ell \neq 0$,

$$
\omega=k U+i U_{y} \frac{k}{\ell}
$$

This result could suggest - incorrectly - that (5.1) has modes with unbounded growth rates, and hence is ill-posed. It is well-known, however, that the well-posedness of a variable coefficient PDE and the corresponding frozen PDEs need not agree when one of them is weakly well-posed (see e.g. [19]). 
(a)

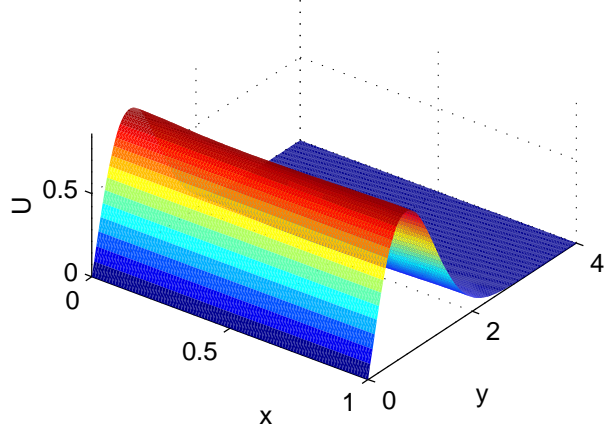

(c)

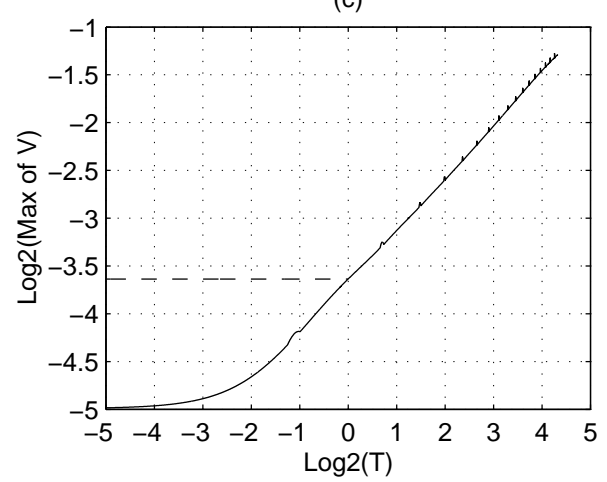

(b)

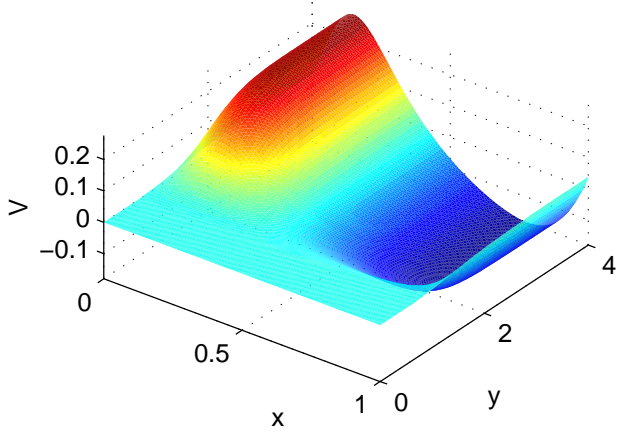

(d)

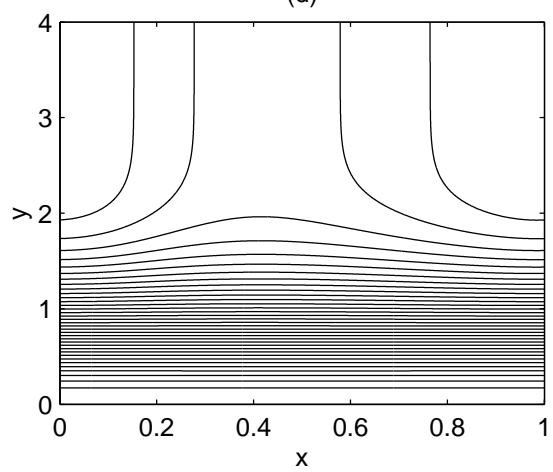

FIG. 6.1. A numerical solution of the inviscid Prandtl equations (2.1)-(2.4), with the initial data $u_{0}(x, y)=U(y)[1+0.005 \sin (2 \pi x)]$, where $U(y)=2 y e^{-y^{2}}:$ (a) a surface plot of $u(x, y, t)$ at time $t=10$; (b) a surface plot of $v(x, y, t)$ at time $t=10$; (c) a log-log plot of the maximum norm $\|v\|_{\infty}$ of $v$ versus $t ;(d)$ streamlines at $t=10$.

In the special case of the exact solution (3.2)-(3.3) of the the inviscid Prandtl equations, we have, for small $\beta$, that

$$
u(x, y, t)=U(y)+U_{y}(y) \beta(x, t)+O\left(\beta^{2}\right) .
$$

Thus, the initial perturbation of $u=U(y)$ is $u_{0}(x, y)=U_{y}(y) \beta_{0}(x)$. Since $U_{y}(c)=0$, we have $u_{0}(x, c)=0$, and the coefficient of the algebraically growing term in (6.4) vanishes, consistent with the lack of growth in the exact solution.

To compare this linearized analysis with the behavior of the nonlinear equations, we computed a numerical solution of the inviscid Prandtl equations (2.1)-(2.4) with initial data corresponding to a sinusoidal perturbation of a shear flow

$$
u_{0}(x, y)=U(y)[1+\epsilon \sin 2 \pi x],
$$

where $\epsilon=0.005$, and

$$
U(y)=2 y e^{-y^{2}}
$$

This profile has a maximum at $y=c$, where $c=1 / \sqrt{2}$. 
Using the above analysis, we find that the time-asymptotic behavior of the solution for $v$ of the linearized equations as $t \rightarrow \infty$ is

$$
v(x, y, t) \sim 2 \epsilon \pi^{2}\left(\frac{2}{e}\right)^{1 / 4} \sqrt{t} V(y) \sin (2 \pi[x-U(c) t]+\pi / 4),
$$

where

$$
V(y)= \begin{cases}U(y)-U(c) & \text { if } y>c \\ 0 & \text { if } 0 \leq y \leq c\end{cases}
$$

The solution of the linearized equations for the perturbed velocity $v$ remains bounded as $t \rightarrow \infty$ when $y<c$, since there is no stationary phase point in $[0, y]$, but we omit a detailed expression for its behavior. It follows from (6.6) that the $L^{\infty}$-norm of $v$ with respect to $(x, y)$ at time $t$ has the asymptotic behavior

$$
\|v\|_{\infty}(t) \sim C t^{1 / 2} \quad \text { as } t \rightarrow \infty,
$$

where

$$
C=\epsilon \pi^{2} 2^{7 / 4} e^{-3 / 4} \approx 0.0784
$$

In Figure 6.1, we show a numerical solution of the inviscid Prandtl equations (2.1)-(2.4) for the initial data (6.5), and periodic boundary conditions in $x$. The solution for $v$ at time $t=10$ is in good qualitative agreement with the large-time asymptotic solution of the linearized equations in (6.6). The instability consists of a growing recirculation of fluid that enters from $y=+\infty$, turns around, and leaves to $y=+\infty$.

In the numerical solution, we see a power law dependence of the maximum norm of $v$ on $t$ over a range of times $1 \leq t \leq 20$. From the numerical data, we estimate

$$
\|v\|_{\infty}(t) \sim A t^{\alpha} \quad \text { where } A \approx 0.080, \alpha \approx 0.51
$$

in good quantitative agreement with the results of the linearized analysis.

After longer times, the linearized approximation breaks down, and $v$ grows more rapidly, eventually appearing to develop a singularity similar to the one illustrated in Section 3.

Finally, we consider the effect of viscosity on this instability. By analogy with what is often done in studying the stability of shear flows in fluid mechanics [11], one could make a "quasi-parallel" assumption and linearize the viscous Prandtl equations with small viscosity about an arbitrary inviscid shear flow solution. The effect of the singular viscous perturbation on the continuous spectrum of the inviscid problem seems difficult to analyze, however, and the errors caused by the quasi-parallel assumption are hard to estimate.

Instead, in Figure 6.2, we show a numerical solution of the viscous Prandtl equations with $\nu=0.1$, and the same initial data as for the inviscid solution shown in Figure 6.1. The resulting solution is a perturbation of the exact diffusing shear flow solution given by (4.3), (4.6). The diffusion of the shear profile in Figure 6.2, from a width of aproximately 2 at $t=0$ to a width of approximately 5 at $t=10$, is significant. Nevertheless, we see a similar instability to the inviscid one. In fact, the growth of $v$ is faster than in the inviscid case. 
(a)

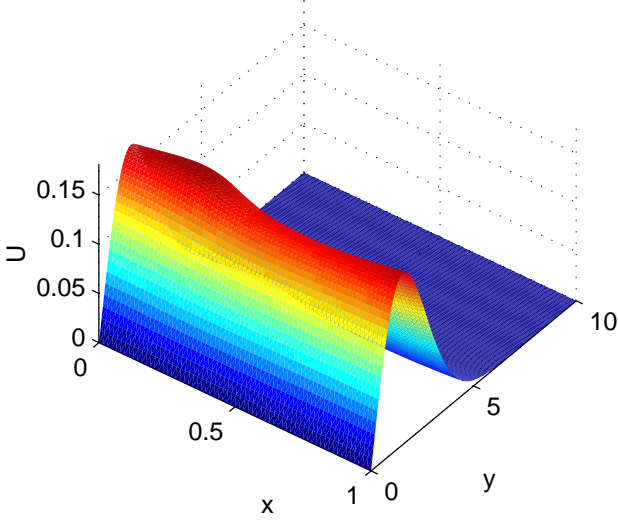

(c)

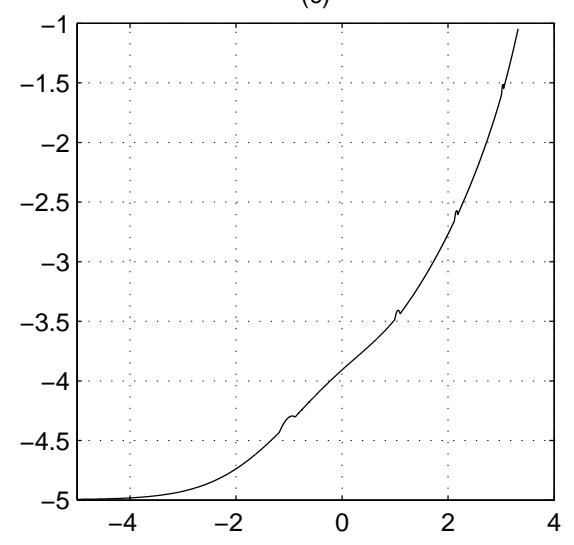

(b)

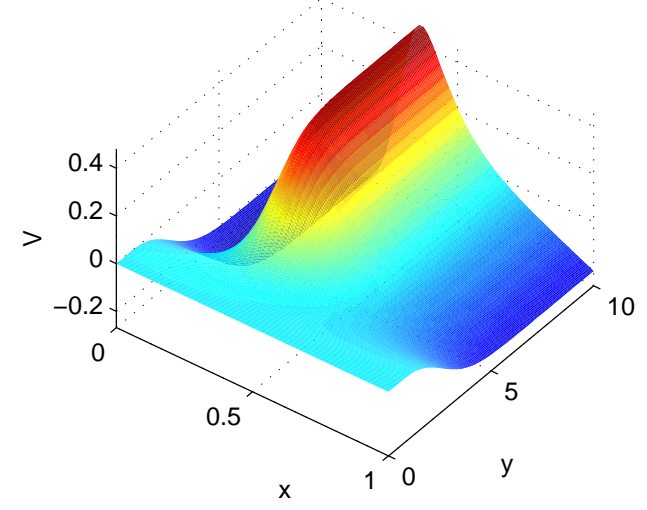

(d)

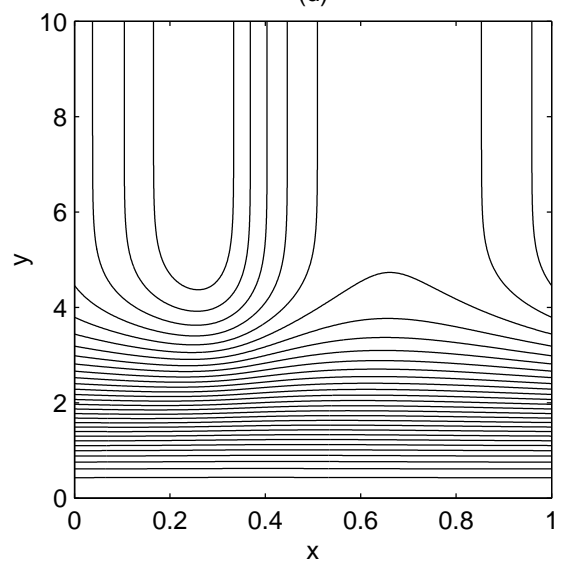

FIG. 6.2. A numerical solution of the viscous Prandtl equations (1.1), with $u^{\infty}=0$ and the initial data $u_{0}(x, y)=U(y)[1+0.005 \sin (2 \pi x)]$, where $U(y)=2 y e^{-y^{2}}:$ (a) a surface plot of $u(x, y, t)$ at time $t=10$; (b) a surface plot of $v(x, y, t)$ at time $t=10$; (c) a log-log plot of the maximum norm $\|v\|_{\infty}$ of $v$ versus $t ;(d)$ streamlines at $t=10$.

\section{Conclusion}

We have solved the inviscid Prandtl equations by the method of characteristics, presented a simple explicit solution that forms a singularity in finite time, and shown that the same type of singularity appears to occur in solutions of the viscous Prandtl equations. Once singularites form, we see a similar phenomenon to the delta-shocks that arise in solutions of some hyperbolic conservation laws.

We have also explicitly solved the linearization of the inviscid Prandtl equations about a shear flow. The resulting equations are weakly, but not strongly, well-posed, and have a continuous spectrum which is unstable when the unperturbed shear flow profile has a critical point. This behavior contrasts with that of the Euler equations, whose linearization about a shear flow has a stable continuous spectrum.

Acknowledgements. We thank Liqun Zhang for helpful discussions. 


\section{REFERENCES}

[1] Y. Brenier, Homogeneous hydrostatic flows with convex velocity profiles. Nonlinearity, 12:495-512, 1999.

[2] K.W. Brinckman and J.D.A. Walker, Instability in a viscous flow driven by streamwise vortices. J. Fluid Mech., 432:127-166, 2001.

[3] R.E. Caflisch and M. Sammartino, Existence and singularities for the Prandtl boundary layer equations. Z. Angew. Math. Mech., 80:733-744, 2000.

[4] K.M. Case, Stability of inviscid plane Couette flow. Phys. Fluids., 3:143-148, 1960.

[5] S.J. Cowley, Computer extension and analytic continuation of Blasius' expansion for impulsive flow past a circular cylinder. J. Fluid Mech., 135:389-405, 1983.

[6] S.J. Cowley, Laminar boundary layer theory: A 20th century paradox?, in Proceedings of ICTAM 2000. eds. H. Aref and J.W. Phillips, Kluwer, 389-411, 2001.

[7] S.J. Cowley, L.L.van Dommelen, and T.T. Lam, On the use of lagrangian variables in descriptions of unsteady boundary-layer separation. Phil. Trans. R. Soc. Lond., 333(A):343-378, 1990.

[8] L.A. Dikii, The stability of plane parallel flows of an ideal fluid. Sov. Phys. Dokl., 5:1179$1182,1960$.

[9] L.L.van Dommelen, Unsteady Boundary Separation. Ph.D. thesis, Cornell University, Ithaca, NY, 1981.

[10] L.L. van Dommelen and S.F. Shen, The spontaneous generation of the singularity in a separating laminar boundary layer. J. Comp. Phys., 38:125-140, 1980.

[11] P.G. Drazin and W.H. Reid, Hydrodynamic Stability. Cambridge University Press, Cambridge, 1981.

[12] W. E and B. Engquist, Blowup of solutions to the unsteady Prandtl's equation. Comm. Pure Appl. Math., 50:1287-1293, 1997.

[13] W. E, Boundary layer theory and the zero-viscosity limit of the Navier-Stokes equation, Acta Math. Sin., 16:207-218, 2000.

[14] S. Friedlander, W. Strauss, and M. Vishik, Nonlinear instability in an ideal fluid. Ann. Inst. Henri Poincaré., 14:187-209, 1997.

[15] E. Grenier, On the derivation of homogeneous hydrostatic equations. Math. Model. Num. Anal., 33:965-970, 1999.

[16] E. Grenier, On the nonlinear instability of Euler and Prandtl equations. Comm. Pure Appl. Math., 53:1067-1091, 2000.

[17] L. Hong, A Numerical and Analytical Study of the Prandtl Equations. Ph.D. Thesis, University of California, Davis, 2002.

[18] B.L. Keyfitz and H.C. Kranzer, A viscosity approximation to systems of conservation laws admitting singular shocks. in Nonlinear Hyperbolic Problems, Lecture Notes in Mathematics, Springer-Verlag, Berlin, Vol.1042, 1989.

[19] H.O. Kreiss, Numerical Methods for Solving Time-Dependent Problems for Partial Differential Equations. Séminaire de Mathématiques Supérieures Départment de Mathématiques et de Statistique Université de Montréal, 1978.

[20] D.K. Ludlow, P.A. Clarkson, and A.P. Bassom, New similarity solutions of the unsteady incompressible boundary-layer equations. Quart. J. Mech. Appl. Math., 53:175-206, 2000.

[21] P.K.H. Ma and W.H. Hui, Similarity solutions of the two-dimensional unsteady boundary layer equations. J. Fluid Mech., 216:537-559, 1990.

[22] O.A. Oleinik, On the mathematical theory of boundary layer for an unsteady flow of incompressible fluid. J. Appl. Math. Mech., 30:951-974, 1966.

[23] O.A. Oleinik and V.N. Samokhin, Mathematical Models in Boundary Layer Theory. Chapman \& Hall/CRC Press, New York, 1999.

[24] G. Puppo, A vortex-grid method for Prandtl's equations. SIAM J. Sci. Comput., 20:12291251, 1999.

[25] S.I. Rosencrans and D.H. Sattinger, On the spectrum of an operator occuring in the theory of hydrodynamics stability. J. Math. Phys., 45:289-300, 1966.

[26] M. Sammartino and R.E. Caflisch, Zero viscosity limit for analytic solutions of the NavierStokes equation on a half-space. I: Existence for Euler and Prandtl equations. Comm. Math. Phys., 192:433-461, 1998.

[27] M. Sammartino and R.E. Caflisch, Zero viscosity limit for analytic solutions of the NavierStokes equation on a half-space. II: Construction of the Navier-Stokes solution. Comm. Math. Phys., 192:463-491, 1998. 
[28] I.J. Sobey, Introduction to Interactive Boundary Layer Theory. Oxford University Press, Oxford, 2000.

[29] V.V. Sychev, A.I. Ruban, V.V. Sychev, and G.L. Korolev, Asymptotic Theory of Separated Flows. Cambridge University Press, Cambridge, 1998.

[30] D. Tan, T. hang, and Y. Zheng, Delta-shock waves as limits of vanishing viscosity for hyperbolic systems of conservation laws. J. Diff. Eq., 112:1-32, 1994.

[31] M. Vishik, Spectrum of small oscillations of an ideal fluid and Lyapunov exponents. J. Math. Pures Appl., 75:531-557, 1996.

[32] Z. Xin and L. Zhang, On the global existence of solutions to the Prandtl's system. Research Report 2002-02(086), The Institute of Mathematical Sciences, The Chinese University of Hong Kong. 